\title{
Coherent control of two photon fluorescence with a high-resolution spectral phase shaper
}

\author{
S. Postma ${ }^{1}$, H.L. Offerhaus ${ }^{1}$, V. Subramaniam ${ }^{2}$, and N.F. van Hulst ${ }^{3}$
}

1 Optical Techniques group, MESA+ Institute for Nanotechnology, Faculty of Science and Technology, University of Twente, P.O. Box 217, 7500 AE Enschede, the Netherlands E-mail: s.postma@utwente.nl

${ }^{2}$ Biophysical Engineering group, MESA+ Institute for Nanotechnology, Faculty of Science and Technology, University of Twente, P.O. Box 217, 7500 AE Enschede, the Netherlands

E-mail: s.postma@utwente.nl

${ }^{3}$ ICFO Institut de Ciències Fotòniques, Av. Canal Olímpic, 08860 Castelldefels, Barcelona, Spain

\begin{abstract}
We present effects of spectral shaping on the two-photon fluorescence from fluorescent dye molecules in solution and from quantum dots. The experiments are done with sub-25 fs pulses around $800 \mathrm{~nm}$ and a compact $\left(7 \times 10 \mathrm{~cm}^{2}\right)$ high resolution reflective spectral phase shaper. Our results demonstrate that the sign of a phase step in the spectrum of the excitation pulses influences the amount of fluorescence. This implies that beside the two-photon spectral intensity the phase of this spectrum is important as well.
\end{abstract}

\section{Introduction}

Optical pulse shaping has a range of interesting possibilities from coherent control of gas-phase diatomic molecules to the selective excitation of large (pathogenic) proteins [1] The presented work demonstrates that the phase of the second harmonic spectrum is of importance for two photon fluorescence.

\section{Spectral phase shaper}

The 'miniature' spectral phase shaper that we have used for these experiments has been described in detail elsewhere [2]. Here we will briefly summarize the relevant aspects of this setup,. Figure 1 shows a schematic and a photograph of the spectral phase shaper. The shaper has a footprint of only $7 \times 10 \mathrm{~cm}^{2}$. The LCM has 4096 elements (Boulder Nonlinear, $1 \mu \mathrm{m}$ by $6 \mathrm{~mm}$ with $1.8 \mu \mathrm{m}$ spacing), yielding more than 600 independent degrees of freedom. The dimensions of the components are chosen such that the crosstalk size of 7 pixels on the array is comparable to the single-color focal spot along the array $(5 \mu \mathrm{m})$. The cross talk between the pixels has been intentionally increased to increase the spatial filling factor. It also reduces pixilation effects [3] and averages out differences in pixel response. 

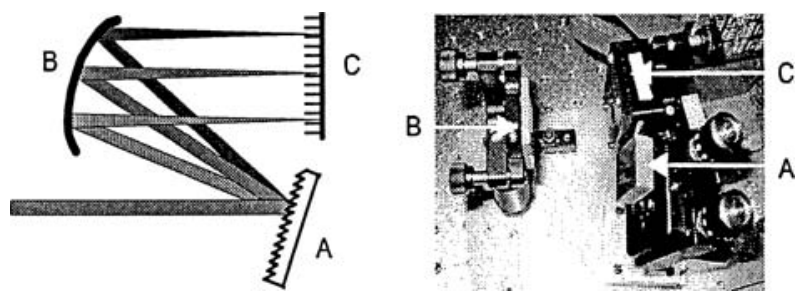

Fig. 1. Schematic and photograph of the spectral phase shaper. (A) grating, (B) cylindrical mirror, (C) liquid crystal modulator.

\section{Results}

In figure 2 we present calculations of the effects of a positive and a negative phase step in the fundamental spectrum on the second harmonic (two-photon) spectrum and spectral phase. Also shown are reference measurements of the signal from an LED (with a gap exceeding the one-photon level, so sensitive only to two photon absorption) for various phase steps, where the horizontal axis indicates the position of the phase step in the spectrum. As can be seen from figure 2(a) and (b), a positive and negative phase step result in the same second harmonic spectrum but a different spectral phase. Figure 2(c) demonstrates that the signal from a two photon absorption LED (a non-resonant transition) is insensitive to the sign of the phase step in the fundamental spectrum. Thus a two photon absorption LED is insensitive for the spectral phase of the second harmonic, as expected.

(a)
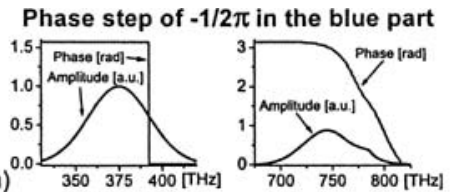

Phase step of $1 / 2 \pi$ in the blue part
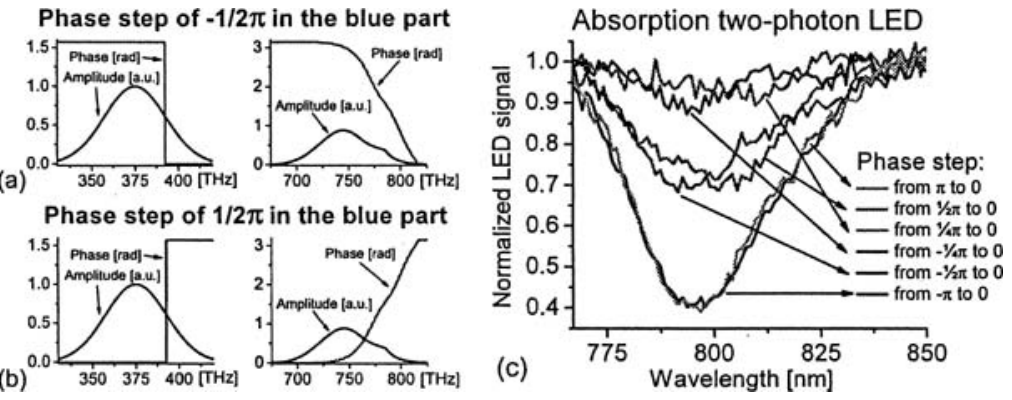

Fig. 2. (a) and (b) calculations of the spectral amplitude and phase of the second harmonic for (a) a negative phase step of $1 / 2 \pi$ at $392.5 \mathrm{THz}$ and (b) for a positive phase step of $1 / 2 \pi$ at $392.5 \mathrm{THz}$. (c) signal of a two photon absorption LED for different amplitudes of the phase steps $(\pi, 1 / 2 \pi, 1 / 4 \pi,-1 / 4 \pi,-1 / 2 \pi,-\pi)$ tuned across the fundamental spectrum. 
Next, we present two photon fluorescence measurements on resonant transitions in Figure3. Figure 3(a) is for the fluorescent dye cascade blue, solved in water, figure 3(b) for quantum dots dried from suspension on a glass cover slide. Contrary to the LED-measurements, we observe a clear difference between a positive and a negative phase step for both the fluorescent dye and the quantum dots. Furthermore, the signal increases above the Fourier limited case for example for phase steps at a wavelength of $777 \mathrm{~nm}$ and $825 \mathrm{~nm}$ and the phase steps of a $1 / 4 \pi$ and $1 / 2 \pi$.
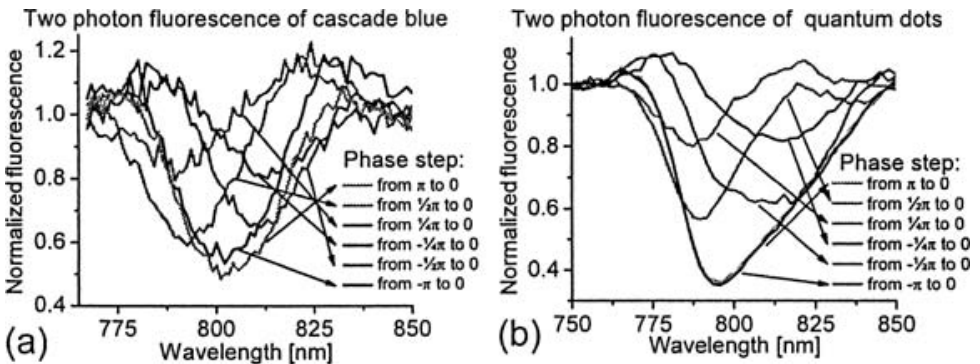

Fig. 3. (a) two photon fluorescence for cascade blue dye for the different phase steps. (b) two photon fluorescence for quantum dots also for the different phase steps.

\section{Conclusions}

We have demonstrated spectral phase shape effects in two photon resonant transitions in the fluorescent dye cascade blue and quantum dots. The shaped resonant transitions show responses exceeding the response for a Fourier limited pulse. A non-resonant transition (LED) shows no such increase, and is insensitive to the sign of the phase step.

\section{References}

1 V.V. Lozovoy and M. Dantus, in ChemPhysChem, Vol. 6, 1971, 2005.

2 S. Postma, H.L. Offerhaus, P. van der Walle, and N.F. van Hulst, in Rev. Sci. Instrum., Vol 76, 123105, 2005.

3 H. Wang, Z. Zheng, D. E. Leaird, A. M. Weiner, T. A. Dorschner, J. J. Fijol, L. J. Friedman, H. Q. Nguyen, and L. A. Palmaccio, in IEEE J. Quantum Electronics, Vol 7, 718, 2001. 